

Research Article

\title{
Effect of magnetite nanoparticles on couple stress fluid between two parallel squeezing and expanding surfaces
}

\author{
Mahesh Kumar $^{1}$ (D) $\cdot$ K. Pravin Kashyap ${ }^{2} \cdot$ N. Naresh Kumar ${ }^{3}$
}

Received: 8 August 2019 / Accepted: 3 December 2019 / Published online: 8 April 2020

(c) Springer Nature Switzerland AG 2020

\begin{abstract}
This article investigates the analysis of hydromagnetic flow of a couple stress fluid through two parallel squeezing surfaces where magnetite nanoparticles are suspended in it. Here ethylene glycol is considered as the base fluid. The dissipations due to magnetic field, viscous and couple stress fluid are considered in the energy equation. The modelled governing differential equations are transformed into dimensionless form of nonlinear coupled ordinary differential equations with the aid of similarity transformations. A shooting method with Runge-Kutta 4th order is utilized to solve the system of reduced flow field equations. The influence of various parameters on the velocity (axial and radial) components and temperature are studied for squeezing and expanding cases for distinct physical parameter through graphs. The results indicate that the axial velocity and temperature show increasing trend for augmenti values of couple stress fluid parameter. Further, radial velocity and temperature also depict augmenting trend for all increasing values of Hartmann number.
\end{abstract}

Keywords Couple stress fluid · Squeezing flow · Ethylene glycol · Magnetite nanoparticles · Shooting method

PACS Nos. 47.15.-x 4 47.10.ad $\cdot 44.90 .+c \cdot 47.60 .+\mathrm{i} \cdot 47.65 .-\mathrm{d} \cdot 02.60 . \mathrm{cb}$

\section{Introduction}

Nowadays the analysis of nanofluid research is a popular area and this interest is escalating due to their essential applicability in modern industries. One of the major complications in improving the heat transfer of industrial systems; for example, electronic cooling and solar stations, is the restriction over the thermal properties of the cooling fluids. The conventional base fluids such as water, ethylene glycol, engine oil, mineral oils and glycerol have less thermal conductivity and they alone are insufficient for achieving requirements of high cooling rate. To solve these kinds of problems, the pioneers like Choi and Eastman [1] introduced new category of fluids and termed as 'nanofluid'. Nanofluids are synthesized by dispersing solid nanoparticles in the base fluids. These fluids have more thermal conductivity and heat transfer efficiency due to the suspension of nanometer-sized particles in the abovementioned conventional fluids [2, 3]. Some nanometersized particles, such as metals ( $\mathrm{Au}, \mathrm{Cu}, \mathrm{Ag})$, carbide ceramics, carbon oxide ceramics $\left(\mathrm{Al}_{2} \mathrm{O}_{3}, \mathrm{CuO}\right.$ ), metal nitrides (AIN, $\mathrm{SiN}), \mathrm{TiO}_{2}, \mathrm{Fe}$, and $\mathrm{SiO}_{2}$, can be used as main components of nanofluid due to its applicability in medical and engineering field [4]. The use of nanofluids in some industrial applications like engines cooling systems, magnetic field sensors, nuclear reactors, magnetic storage media, transportation, and the cooling systems of electronics, could be a promising solution in attaining the required heat transfer capabilities. This improved thermal performance of nanofluids can give a betterment in a number of

$\triangle$ N. Naresh Kumar, nareshkumarn622@gmail.com | 'Department of Mathematics, Central University of Karnataka, Kalaburagi, India. ${ }^{2}$ Department of Applied Mathematics, Defence Institute of Advanced Technology (Deemed University), Pune, Maharashtra 411025, India. ${ }^{3}$ Department of Mathematics, SASTRA Deemed University, Thanjavur, Tamil Nadu 613401, India. 
industrialized sectors including fuel cells, transportation, microelectronics, micro-manufacturing, pharmaceutical processes, chemical and metallurgical sectors, along with cancer therapy and it is also a safer option in surgery by cooling, biomedicine, and many more [5-7]. However, in the literature there are various number of nanoparticles and base fluids have been explored to analyse different characteristics of nanofluids. Among those $\mathrm{Fe}_{3} \mathrm{O}_{4}$ nanofluids, is an essential one and it has been extensively studied and applied into various fields of research. $\mathrm{Fe}_{3} \mathrm{O}_{4}$ nanofluid has the capability to retort to an external applied magnetic field and it reveals magnetic and liquid properties that are developed in a diverse of applications in cooling systems of micro devices [8].

The heat transfer study of time-dependent squeezing flow between parallel plates is one among utmost significant research areas owing to its widespread in engineering and scientific applications, such as power transmission systems, aerodynamic heating, compression, polymer processing, modelling the lubrication equipment, hydrodynamical machines, injection moulding, animal joints, bio-lubrication joints, destruction of crops caused by freezing, synovial joints, thrust bearing, chemical processing, automotive components, lubrication system etc. These squeezing flows are made by employing vertical velocities or normal stresses via moving boundary, which could lead to different hydro-dynamical technologies and tools. The earliest analysis on the squeezing flow was described by Stefan [9]. Following Stefan, many investigators have considered squeezing flow concept for theoretical and experimental study [10-18]. These investigations are restricted to Newtonian fluid. However, in the evolution of modern engineering, the role of non-Newtonian squeeze-flow is vital. Numerous scholars have analysed the squeezing flow problem with various non-Newtonian fluid models [19-25]. Amongst of these non-Newtonian models, couple stress fluid model proposed by Stoke's [26] has been given considerable attention by various investigators in field of fluid mechanics. This model sufficiently describes the fluidflow behaviour of some polymer additives, animal blood and liquid crystals substructure. The stresses in this fluid model are formed due to mechanical interaction of in the medium of fluid. Colloidal fluids, blood, polymer thickened oils, polymer suspensions, synthetic fluids, liquid crystals, lubrications and additive electro-rheological fluids, etc. are some of the examples and importance of this fluid model can be widely seen in the analysing axisymmetric flow problems [27], lubrication theory [28], thin film [29], peristaltic transport [30], squeeze film [31] and many more.

Another topic portrayed in this article is the analysis of magnetohydrodynamic (MHD). This theory explores dynamics of electrically conducting fluids, for instance, salt water, molten metal liquid metals, and plasma. MHD is a flux concept which can be expressed in terms of parameters of flow-field variables, pressure, density and applied magnetic fields. MHD spectroscopy, microfluidic devices, electromagnetic stirring, Jet printer, MHD pump, microelectronics, MHD generators, MHD dynamo and electromagnetic valves, etc. are some of the applications. Also, the squeezing flow with MHD effect leads to significant applications particularly in the field of chemical industry, liquid-metal lubrications, and fusion reactors for cooling of nuclear reactor, etc.,. The effect of MHD on squeezing flow for various geometries and explored the importance of these flows [32-38]. Recently, Qayyum et al. [39] examined the MHD squeezing flow of non-Newtonian fluid in a porous medium. Ghadikolaei et al. [40] investigated the magnetohydrodynamic squeezing flow, heat and mass transfer of Eyring-Powell fluid between two parallel plates. Muhammad et al. [41] analysed the hydromagnetic transient squeezing flow of viscoelastic fluid between walls. Similar unsteady MHD squeezing flow problem for radiative non-Newtonian fluid in a channel was examined by Adesanya [42]. Hayat et al. [43] considered the squeezing flow of couple stress fluid in a parallel plate channel where the effective electrical conductivity is not considered. Nagler [44] examined the Jeffrey-Hamel flow of nanofluid inside a wedge analytically. Recently, Ahmad et al. [45] explored the comparative study on MHD squeezing flow problem with entropy generation. He considered water as base fluid with five different nanoparticles. Aforementioned literature shows that no endeavour has been made on time-dependent hydromagnetic flow of couple stress fluid in a squeezing and expanding channel with magnetite $\left(\mathrm{Fe}_{3} \mathrm{O}_{4}\right)$ nanoparticles.

\section{Physical model}

Consider an unsteady 2-D hydromagnetic squeezing flow of couple stress fluid with suspended $\mathrm{Fe}_{3} \mathrm{O}_{4}$ nanoparticles in ethylene glycol in a parallel plate channel as depicted in Fig. 1. The distance between two plates is



Fig. 1 Geometry of the problem 
considered to be function of time $h(t)=\sqrt{\frac{v(1-\gamma t)}{a}}$. The lower plate is considered as permeable stretching surface and upper plate is moving with velocity $\frac{\gamma}{2} \sqrt{\frac{v}{a(1-\gamma t)}}$ towards the lower plate. The fluid flow is presumed to be incompressible and it is electrically conductive in nature. The presence time dependent magnetic field $\frac{B_{0}}{(1-\gamma t)}$ which applied in the Z-direction has an effect on the momentum and temperature across the channel. The governing conservation equations are given by

$\frac{\partial u}{\partial x}+\frac{\partial v}{\partial y}=0$

$\rho_{n f}\left(u \frac{\partial u}{\partial x}+v \frac{\partial u}{\partial y}+\frac{\partial u}{\partial t}\right)=-\frac{\partial p}{\partial x}+\mu_{n f} \nabla^{2} u-\eta_{1} \nabla^{4} u-\frac{\sigma_{n f} B_{0}^{2} u}{(1-\gamma t)}$

$\rho_{n f}\left(u \frac{\partial v}{\partial x}+v \frac{\partial v}{\partial y}+\frac{\partial v}{\partial t}\right)=-\frac{\partial p}{\partial y}+\mu_{n f} \nabla^{2} v-\eta_{1} \nabla^{4} v-\frac{\sigma_{n f} B_{0}^{2} v}{(1-\gamma t)}$

$\rho_{n f} c_{n f}\left(u \frac{\partial T}{\partial x}+v \frac{\partial T}{\partial y}+\frac{\partial T}{\partial t}\right)=k_{n f} \nabla^{2} T+\eta_{1}\left(\left(\nabla^{2} u\right)^{2}+\left(\nabla^{2} v\right)^{2}\right)$

$$
\begin{aligned}
+ & 4 \mu_{n f}\left(\frac{\partial u}{\partial x}\right)^{2}+\mu_{n f}\left(\frac{\partial u}{\partial y}+\frac{\partial v}{\partial x}\right)^{2} \\
& +\frac{\sigma_{n f} B_{0}^{2}\left(u^{2}+v^{2}\right)}{(1-\gamma t)}
\end{aligned}
$$

with associated boundary conditions are given by:

$y=0$ :

$T=T_{0}, v=\frac{-V_{0}}{1-\gamma t}, u=U_{0}=\frac{a x}{1-\gamma t}, \quad \frac{\partial u}{\partial y}=0$

$y=h(t):$

$T=T_{0}+\frac{T_{0}}{1-\gamma t}, v=-\frac{\gamma}{2} \sqrt{\frac{v}{a(1-\gamma t)}}, u=0, \quad \frac{\partial u}{\partial y}=0$

In the above equation $u$ and $v$ are the velocities along the horizontal and vertical directions respectively, $p$ is the pressure, $\mathrm{T}$ is the temperature and $\eta_{1}$ is the couple stress viscosity parameter, $\mathrm{T}_{0}$ denotes lower stretching porous surface temperature, 'a' stands for lower surface stretching rate, $V_{0}>0$ and $V_{0}<0$ shows the suction and injection velocity, respectively. The parameters namely, $\mu_{n f}, \rho_{n f},\left(\rho c_{p}\right)_{n f}, k_{n f}$ and $\sigma_{n f}$ respectively denote nanofluid viscosity, density, specific heat capacity, thermal conductivity and electrical conductivity.

The relation between the viscosity of the nanofluid and the base fluid as given by Brinkman [46]

$\mu_{n f}=\frac{\mu_{f}}{\sqrt{(1-\varphi)^{2.5}}}$

Following Tiwari and Das [47], the effective heat capacity and effective density are expressed as

$\rho_{n f}=(1-\varphi) \rho_{f}+\varphi \rho_{s},(\rho c)_{n f}=(1-\varphi)(\rho c)_{f}+\varphi(\rho c)_{s}$

The effective thermal conductivity of nanofluid as (Maxwell-Garnett model by following Khanafer et al. [48])

$\frac{k_{n f}}{k_{f}}=\frac{k_{s}+2 k_{f}-2 \varphi\left(k_{f}-k_{s}\right)}{k_{s}+2 k_{f}+2 \varphi\left(k_{f}-k_{s}\right)}$

Following Maxwell [49], the effective electrical conductivity of nanofluid is given by

$\frac{\sigma_{n f}}{\sigma_{f}}=1+\frac{3\left(\frac{\sigma_{s}}{\sigma_{f}}-1\right) \varphi}{\frac{\sigma_{s}}{\sigma_{f}}+2-\left(\frac{\sigma_{s}}{\sigma_{f}}-1\right) \varphi}$

$\varphi$ denotes the nanoparticle volume fraction. $\rho_{s}, c_{s}, k_{s}, \sigma_{s}$ and $\rho_{f}, c_{f}, k_{f}, \sigma_{f}$ are density, specific heat, thermal conductivity, electric conductivity of nanoparticles and base fluid respectively.

Introducing following similarity transformations [43] leads to the study in one variable $(\eta)$.

$T=T_{0}+\frac{T_{0}}{1-\gamma t} \theta(\eta), u=U_{0} f^{\prime}(\eta)$,

$v=-\sqrt{\frac{a v}{1-\gamma t}} f(\eta) \quad$ where $\quad \eta=\frac{y}{h(t)}$

Substituting (6)-(10) into (2), (3) and (4), we obtain

$$
\begin{aligned}
f^{v i}= & \frac{1}{\beta(1-\varphi)^{2.5}}\left(f^{i v}-A_{2} H a^{2}(1-\varphi)^{2.5} f^{\prime \prime}\right) \\
& -\frac{A_{1}}{\beta}\left(\frac{S_{q}}{2}\left(3 f^{\prime \prime}+\eta f^{\prime \prime \prime}\right)+f^{\prime} f^{\prime \prime}-f f^{\prime \prime \prime}\right)
\end{aligned}
$$

$$
\begin{aligned}
\theta^{\prime \prime}= & \frac{\operatorname{Pr} A_{3}}{A_{4}}\left(S_{q}\left(\theta+\frac{\eta \theta^{\prime}}{2}\right)-f \theta^{\prime}\right)-\frac{\operatorname{Pr} E c}{A_{4} \sqrt{(1-\varphi)^{5}}}\left(4 f^{\prime 2}+\delta^{2} f^{\prime \prime 2}\right)-\frac{\beta \operatorname{Pr} E C}{A_{4}}\left(f^{\prime \prime 2}+\delta^{2} f^{\prime \prime \prime 2}\right) \\
& -\frac{A_{2} \operatorname{Pr} E c H a^{2}}{A_{4}}\left(f^{2}+\delta^{2} f^{\prime 2}\right)
\end{aligned}
$$


Table 1 Thermophysical properties of base fluids and nanoparticles

\begin{tabular}{lllll}
\hline & $\rho\left(\mathrm{kg} \mathrm{m}^{-3}\right)$ & $c_{p}\left(\mathrm{~J} \mathrm{~kg}^{-1} \mathrm{~K}^{-1}\right)$ & $k\left(\mathrm{~W} \mathrm{~m}^{-1} \mathrm{~K}^{-1}\right)$ & $\sigma(\Omega \mathrm{m})^{-1}$ \\
\hline $\begin{array}{c}\text { Ethylene } \\
\text { glycol }\end{array}$ & 1132 & 2349 & 0.258 & $3.3 \times 10^{-4}$ \\
$\mathrm{Fe}_{3} \mathrm{O}_{4}$ & 5180 & 670 & 9.7 & 25,000 \\
\hline
\end{tabular}

where $S_{q}=\frac{\gamma}{a}$ is the squeezing number which mainly depends on the motion of the plates, $\beta=\frac{\eta_{1} a}{v_{f}^{2} \rho_{f}(1-\gamma t)}$ is the couple stress fluid parameter, $\mathrm{Pr}=\frac{\mu_{f} c_{f}}{k_{f}}$ is Prandtl number, $\mathrm{Ha}^{2}=\frac{\sigma_{f} B_{0}^{2}}{\rho_{f} a}$ is Hartmann number, $E c=\frac{v_{f} a}{c_{f} T_{0}}$ is Eckert number. $A_{1}, A_{2}, A_{3}$ and $A_{4}$ are nanofluid parameters corresponding to density, electrical conductivity, specific heat capacity and they are defined as

$$
\begin{gathered}
A_{1}=(1-\varphi)+\varphi \frac{\rho_{s}}{\rho_{f}}, \quad A_{2}=1+\frac{3\left(\frac{\sigma_{s}}{\sigma_{f}}-1\right) \varphi}{\frac{\sigma_{s}}{\sigma_{f}}+2-\left(\frac{\sigma_{s}}{\sigma_{f}}-1\right) \varphi}, \\
A_{3}=(1-\varphi)+\varphi \frac{(\rho c)_{s}}{(\rho c)_{f}}, A_{4}=\frac{k_{s}+2 k_{f}-2 \varphi\left(k_{f}-k_{s}\right)}{k_{s}+2 k_{f}+2 \varphi\left(k_{f}-k_{s}\right)}
\end{gathered}
$$

The values for $A_{1}, A_{2}, A_{3}$ and $A_{4}$ are computed by using the values in Table 1.

Non-dimensional form of boundary conditions can be written as

$f(0)=S, f^{\prime}(0)=1, f^{\prime \prime}(0)=0, \theta(0)=0$,

$f(1)=\frac{S_{q}}{2}, f^{\prime}(1)=0, f^{\prime \prime}(1)=0, \theta(1)=1$

where $S=\frac{V_{0}}{a h}$ for suction/blowing parameter.

\section{Skin friction}

The shear stress for couple stress fluid is given by

$\tau_{w}=\mu_{n f} \frac{\partial u}{\partial y}-\eta_{1} \frac{\partial^{3} u}{\partial y^{3}}$

The local skin-friction coefficient $C_{f}$ at lower and upper surfaces is given by

$C_{f}=\frac{2 \tau_{w}}{\rho v_{w}^{2}}=\left(\frac{1}{(1-\varphi)^{2.5}} f^{\prime \prime}(\eta)-\beta f^{i v}(\eta)\right)_{\eta=0,1}$

\section{Nusselt number}

The Nusselt number at lower and upper surfaces defined as follows

$$
N u=-\left(\theta^{\prime}(\eta)\right)_{\eta=0,1}
$$

\section{Mathematical solution}

The Eqs. (11) and (12) in $\eta$ are integrated with a shooting technique using the boundary conditions at 0 and 1 . In order to carry the integration, the coupled system of non-linear differential equations is set in a system form by taking $\mathrm{f}^{(\mathrm{i})}(\eta)=\mathrm{y}_{\mathrm{i}+1}(\mathrm{i}=1,2,3,4,5), \theta(\eta)$ and $\theta^{\prime}(\eta)$ are $\mathrm{y}_{7}$ and $\mathrm{y}_{8}$.

$$
\begin{aligned}
y_{6}^{\prime}= & \frac{1}{\beta(1-\varphi)^{2.5}}\left(y_{5}-A_{2} H a^{2}(1-\varphi)^{2.5} y_{3}\right) \\
& -\frac{A_{1}}{\beta}\left(\frac{S_{q}}{2}\left(3 y_{3}+\eta y_{4}\right)+y_{2} y_{3}-y_{1} y_{4}\right) \\
y_{8}^{\prime}= & \frac{\operatorname{Pr} A_{3}}{A_{4}}\left(S_{q}\left(y_{7}+\frac{\eta y_{8}}{2}\right)-y_{1} y_{8}\right)-\frac{\operatorname{Pr} E C}{A_{4} \sqrt{(1-\varphi)^{5}}}\left(4 y_{2}^{2}+\delta^{2} y_{3}^{2}\right) \\
& -\frac{\beta \operatorname{Pr} E C}{A_{4}}\left(y_{3}^{2}+\delta^{2} y_{4}^{2}\right)-\frac{A_{2} \operatorname{Pr} E C H a^{2}}{A_{4}}\left(y_{1}^{2}+\delta^{2} y_{2}^{2}\right)
\end{aligned}
$$

The integrating can be done by any standard numerical procedure. In this study, we have implemented RK4 algorithm (4th order Runge-Kutta method) due to its fast convergence nature in most of the highly non linear cases. But RK4 solves an initial value problem and the system (17) and (18) is a boundary value problem. In order to solve this, additional slopes are assumed at the stretching sheet surface, i.e. at $\eta=0$.

$y_{1}(0)=S, \quad y_{2}(0)=1, \quad y_{3}(0)=0, \quad y_{4}(0)=a_{1}$,

$y_{5}(0)=a_{2}, \quad y_{6}(0)=a_{3}, \quad y_{7}(0)=0, \quad y_{8}(0)=a_{4}$

Boundary conditions

$y_{1}(1)=\frac{S_{q}}{2}, \quad y_{2}(1)=0, \quad y_{3}(1)=0, \quad y_{7}(1)=1$

Now, we enforce these initial values in the 4 th order Runge-Kutta method to compute the values at the upper wall $\eta=1$. The initial values consist four unknowns and the boundary conditions are specified for 4 variables. Thus an algebraic $4 \times 4$ system of nonlinear equations has been formulated. This system is solved by employing a Newton Rapshson method which has a convergence rate 2 .

In order to validate the results, the numerical values of non-dimensional shear stress at lower and upper surfaces, for different values of $\mathrm{S}$ and $\mathrm{Ha}$ for Newtonian fluid are tabulated in the Table 2. From this, one can observe that the present results are very much agreeing with Hayat et al. [43].

Table 2 Comparative values of non-dimensional shear stress at both surfaces for different values of $\mathrm{S}$ and $\mathrm{Ha}$ when $\mathrm{S}_{\mathrm{q}}=0, \beta=0$

\begin{tabular}{lllllll}
\hline $\mathrm{S}$ & Ha & \multicolumn{2}{l}{ Hayat et al. [43] } & \multicolumn{2}{l}{ Present } \\
\cline { 3 - 4 } \cline { 6 - 7 } & & $f^{\prime \prime}(0)$ & $f^{\prime \prime}(1)$ & & $f^{\prime \prime}(0)$ & $f^{\prime \prime}(1)$ \\
\hline 0.5 & 0.0 & -7.411153 & 4.713303 & & -7.411145 & 4.713291 \\
& 1.0 & -7.591618 & 4.739017 & & -7.591607 & 4.739007 \\
& 2.0 & -8.110334 & 4.820251 & & -8.110315 & 4.820231 \\
& 3.0 & -8.910096 & 4.964870 & & -8.910067 & 4.964837 \\
0.0 & 2.0 & -4.587891 & 1.842447 & & -4.587883 & 1.842438 \\
0.3 & & -6.665662 & 3.653695 & & -6.665648 & 3.653682 \\
0.6 & & -8.851444 & 5.391248 & & -8.851422 & 5.391224 \\
1.0 & & -11.948584 & 7.593426 & -11.948548 & 7.593391 \\
\hline
\end{tabular}




\section{Results and discussions}

This section addresses the physical analysis and discussion on $\mathrm{Fe}_{3} \mathrm{O}_{4}$ - ethylene glycol nanofluid velocity $\left(f^{\prime}, f\right)$ and temperature $(\theta)$ profiles in two different situations with respect to the movement of the plates $\left(S_{q}>0\right)$ and $\left(S_{q}<0\right)$. The results are obtained for various physical parameter values such as couple stress parameter $\beta$, Hartmann number $\mathrm{Ha}$, volume fraction $\varphi$ and squeezing parameter $\mathrm{S}_{\mathrm{q}}$.

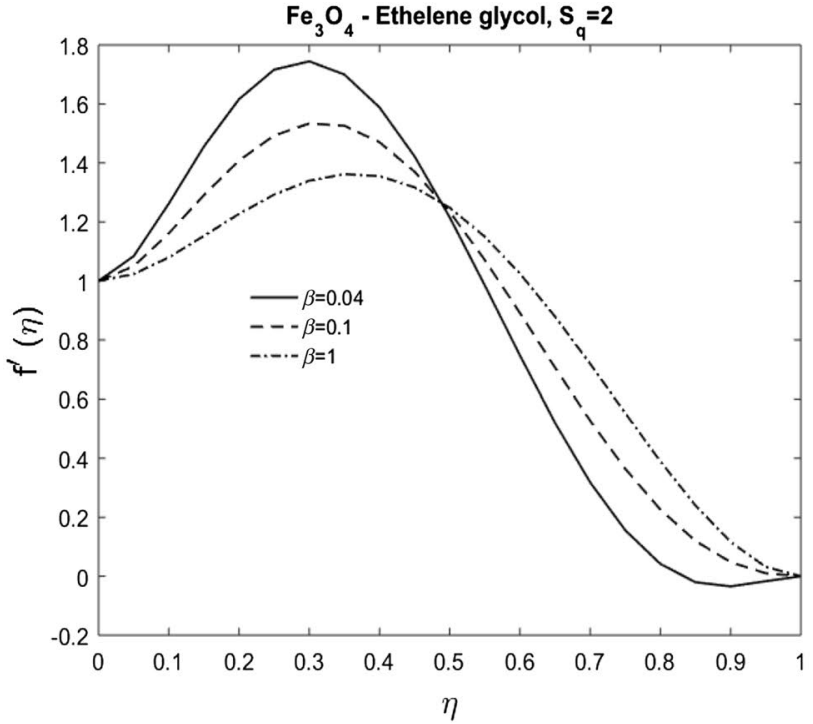

(a)

\subsection{Velocity profile}

The variation of couple stress parameter $(\beta)$ on axial and radial velocity profiles of $\mathrm{Fe}_{3} \mathrm{O}_{4}$-ethylene glycol nanofluid is displayed in Figs. 2 and 3. The positive and negative situations of wall motion have distinct effect on velocity components. Figure $2 \mathrm{a}, \mathrm{b}$ reveal that as $\beta$ augments, the axial velocity shows decreasing trend initially i.e., $0<\eta<0.5$ and a reverse trend for $\eta>0.5$ in both modes of $S_{q}$.

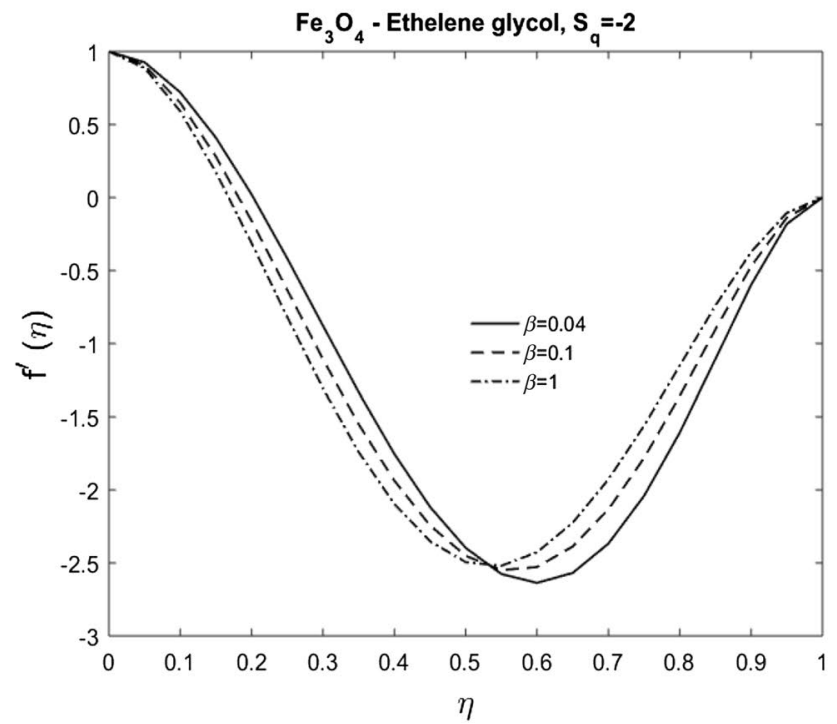

(b)

Fig. 2 Axial velocity profile for different values of couple stress parameter

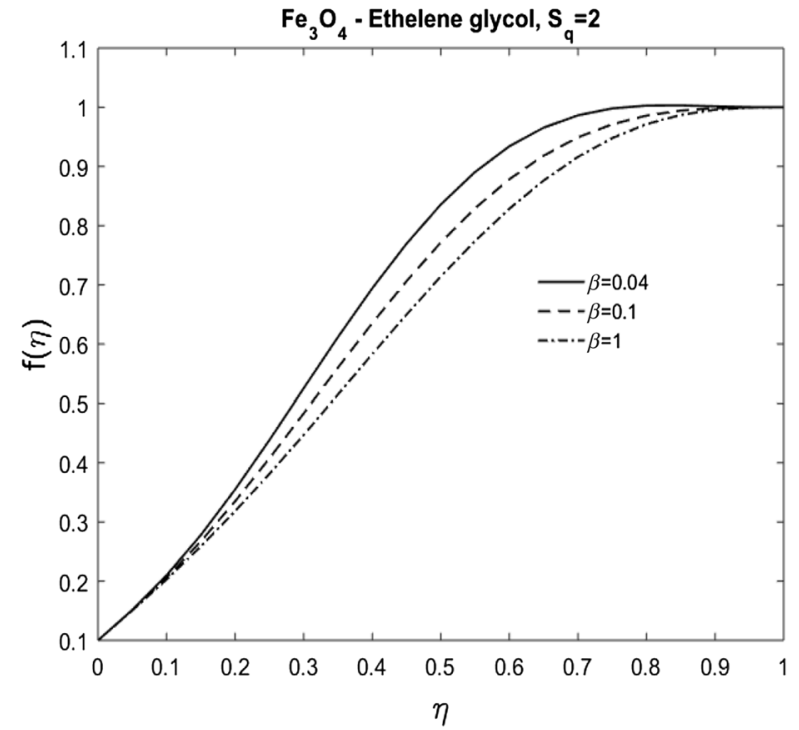

(a)

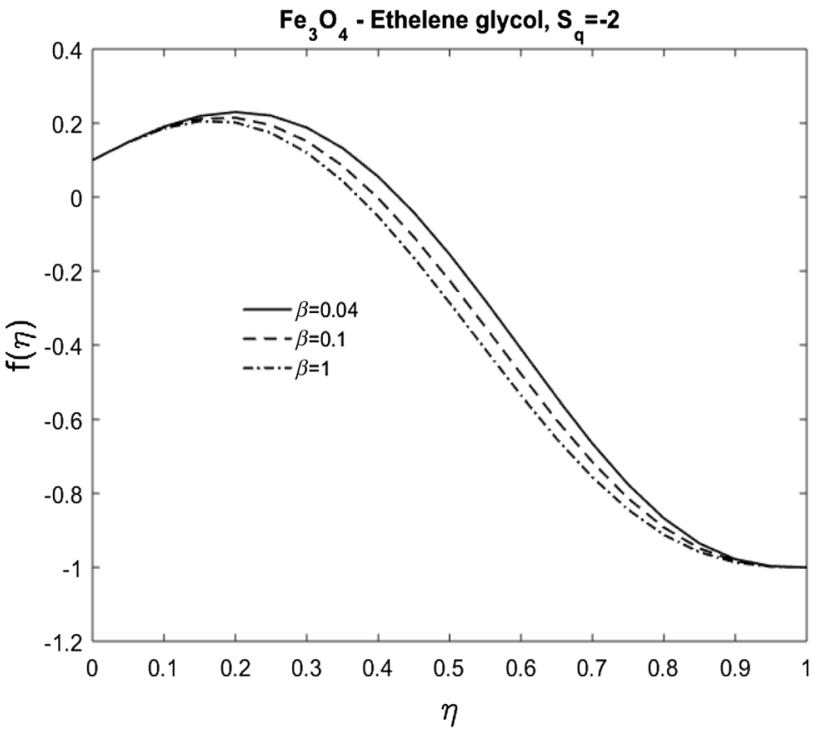

(b)

Fig. 3 Radial velocity profile for different values of couple stress parameter 
Physically, the effect of $S_{q}$ is insignificant in the initial values of eta (the time and space variable). As the upper plate moves in downward direction, an extra force is instigated on the liquid particles which result in a boost in the axial velocity. The radial velocity profile is depicted in Fig. $3 a$, $b$. From Fig. $3 a, b$ it is clear that the radial velocity decreases for increasing $\beta$ values. The reason is obvious, as $\mathrm{S}_{\mathrm{q}}<0$ indicates the upper plate is moving towards lower plate, the plate movement results in a force in the downward direction of the fluid and hence velocity reduces. Similarly, when plate is moving away i.e., $\mathrm{Sq}>0$ the radial velocity of fluid decreases in the negative direction as the volume occupied by the fluid in the channel increases.

Figures 4 and 5 illustrate the influence of Hartmann number $\mathrm{Ha}$ on velocity profiles. Figure $4 \mathrm{a}$, b describes the variation in axial velocity profiles $\left(f^{\prime}\right)$ for different values of $H a$. At the outset, $f^{\prime}$ increases when $0<\eta<0.5$ whereas velocity reduces when $\eta<0.5$ for all augmenting values $\mathrm{Ha}$ and $\mathrm{S}_{\mathrm{q}}>0$. However reverse trend is noticed for the case, $\mathrm{S}_{\mathrm{q}}<0$. Since in a MHD flow, the reduction in the velocity of the fluid at the neighboring surface of the plates would be balanced by an upsurge in the fluid velocity adjacent to the central region and this develops a cross-flow behavior. Figure $5 a, b$ describe the behavior of radial velocity ffor boosting values of $\mathrm{Ha}$. The radial velocity increases in the both cases of wall motion for all augmented values of $\mathrm{Ha}$. This is due to the magnetic resistive forces on the nanoparticles and the base fluid velocity field near the lower sheet.

Figures 6 and 7 depicts the impact of nanoparticle volume fraction $(\varphi)$ on velocity profiles $f^{\prime}$ and $f$. From Fig. 6a, b

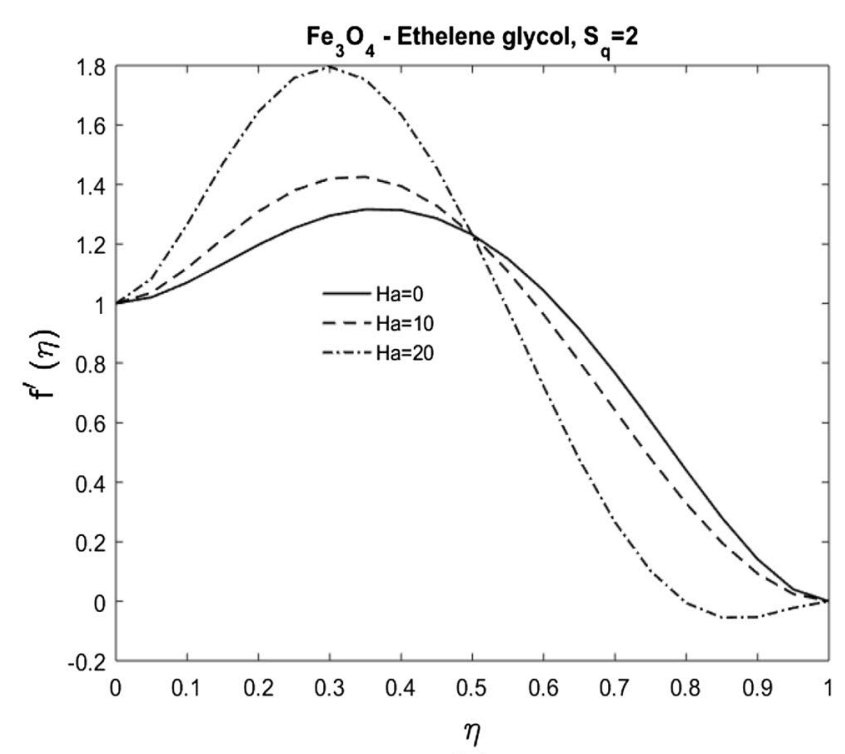

(a) it is observed that axial velocity profile $f^{\prime}$ changes abruptly for augmenting values of $\varphi$. Also, boundary layer thickness increases when $\mathrm{S}_{\mathrm{q}}>0$ whereas it decreases for $\mathrm{S}_{\mathrm{q}}<0$. Similarly, radial velocity fincreases for all augmenting values of $\varphi$ in both mode of $\mathrm{S}_{\mathrm{q}}$ as depicted in Fig. $7 \mathrm{a}$ and $\mathrm{b}$.

\subsection{Temperature profile}

Effect of couple stress parameter ( $\beta$ ), Hartmann number $(\mathrm{Ha})$ and volume fraction $(\varphi)$ on temperature profile $\theta$ are depicted in Fig. 8, 9 and 10. Figure $8 a$, b denotes the variation of $\beta$ values on temperature profile. When $S_{q}>0$, the temperature has a decreasing trend (as thermal boundary increases) for augmented values of $\beta$. Since the movement of upper plate is in downward direction, the friction between the surface of the boundary plate and nanofluid reduces and hence temperature of the couple stress nanofluid decreases. On the other hand, the temperature shows a complete reverse trend for the case $S_{q}<0$. Figure $9 a, b$ represents the temperature profile distribution with respect to $\mathrm{Ha}$. Almost similar trend is observed for all values of $\mathrm{Ha}$ in both cases of $\mathrm{S}_{\mathrm{q}}$. The temperature profile $\theta$ is an increasing function for all augmenting values of $\mathrm{Ha}$. Physically increasing Hartmann number results in Joule heating and thus more heat is created. It is also noted that when $S_{q}>0$, thermal boundary layer thickness is more as compared to $S_{q}<0$. For both the cases of $S_{q}$ and various values of nanoparticle volume fraction, Fig. 10a, b show the effect on temperature profile.

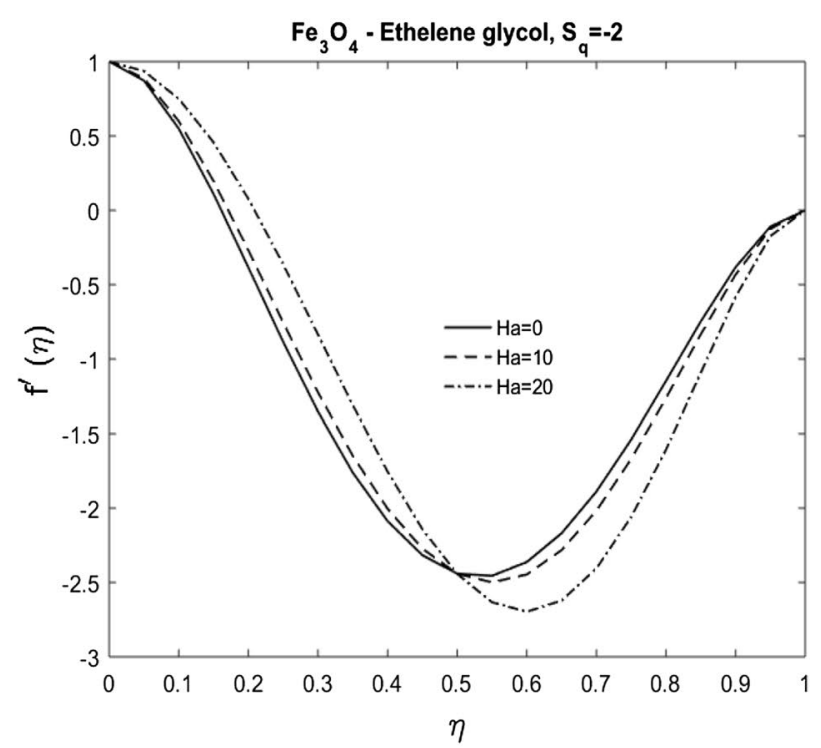

(b)

Fig. 4 Axial velocity profile for different values of Hartmann number 




(a)

Fig. 5 Radial velocity profile for different values of Hartmann number



(a)

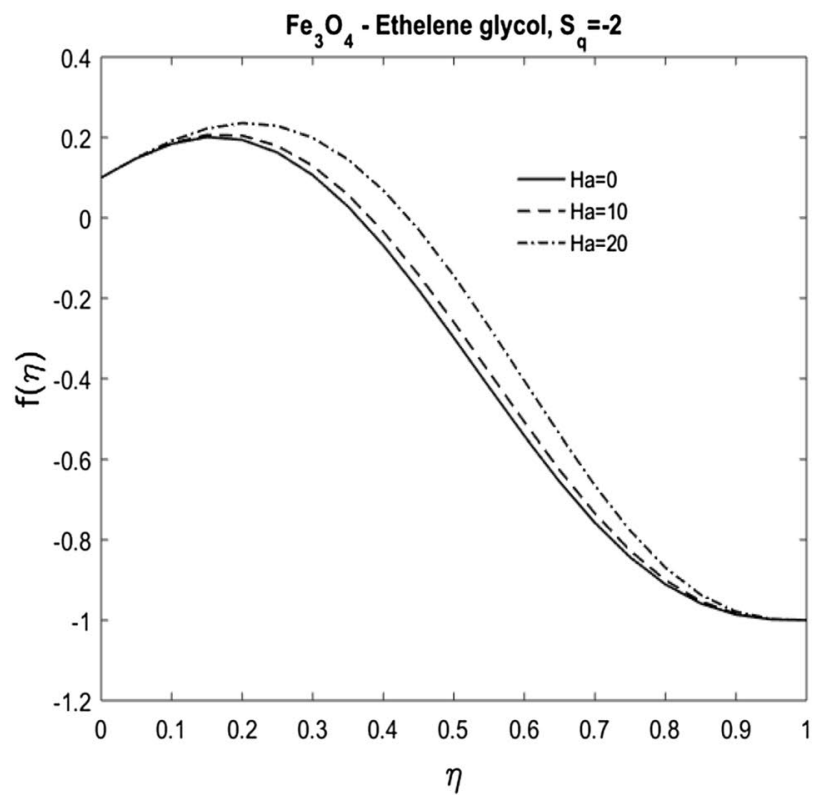

(b)

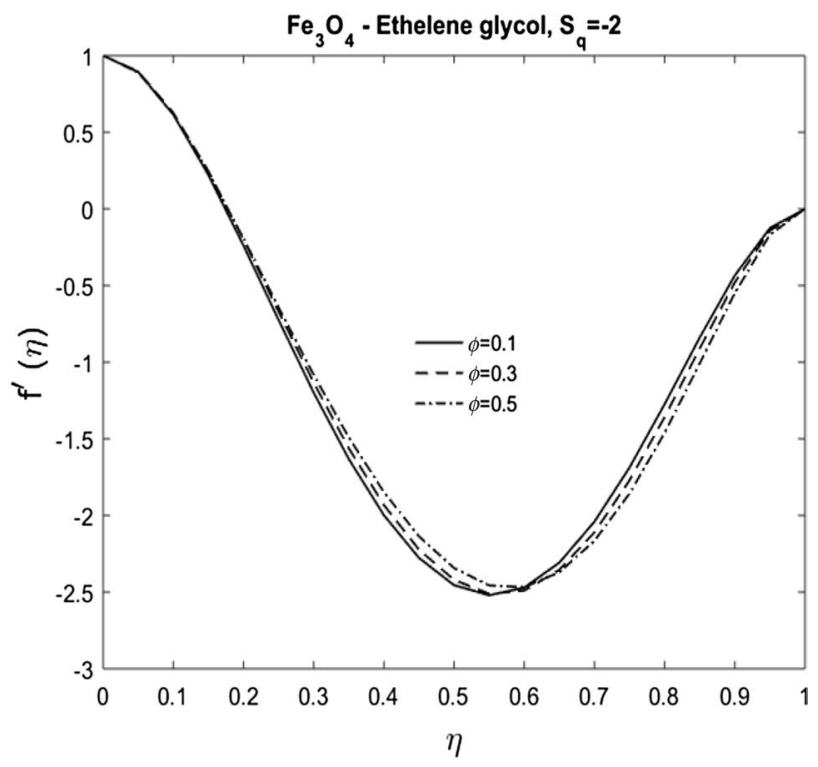

(b)

Fig. 6 Axial velocity profile for different values of volume fraction 


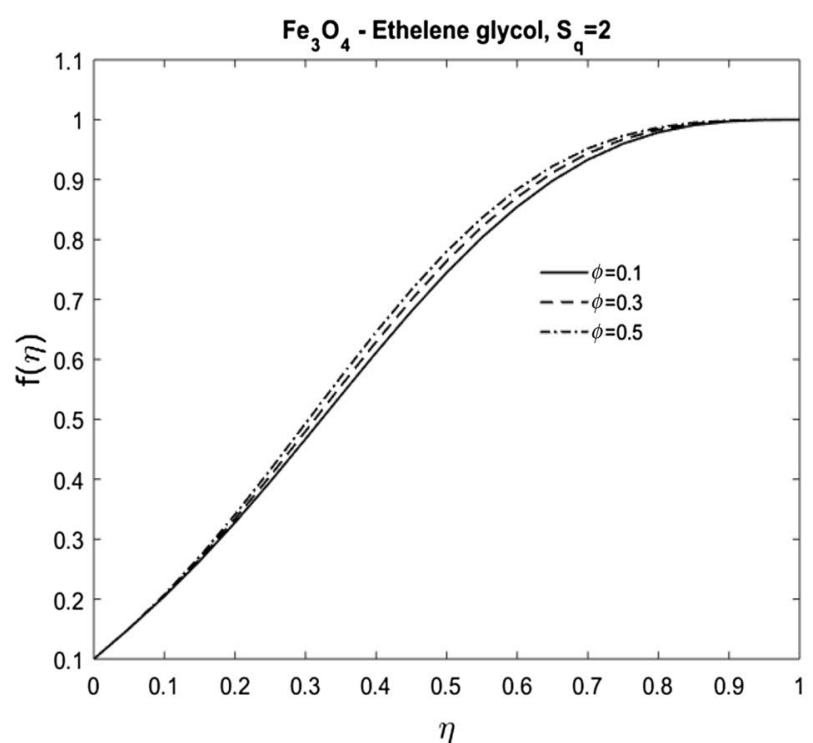

(a)

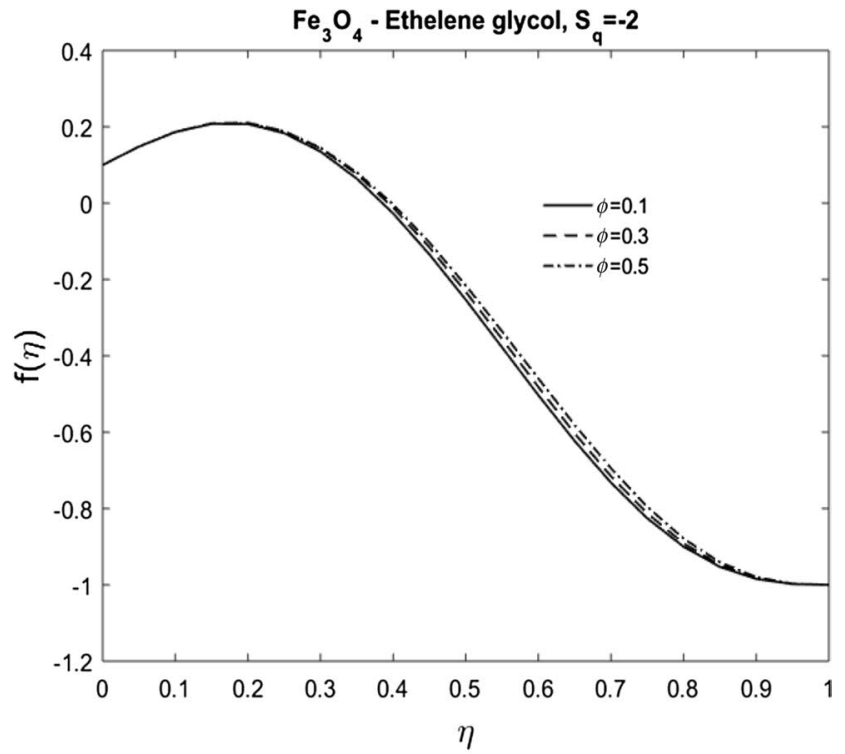

(b)

Fig. 7 Radial velocity profile for different values of volume fraction

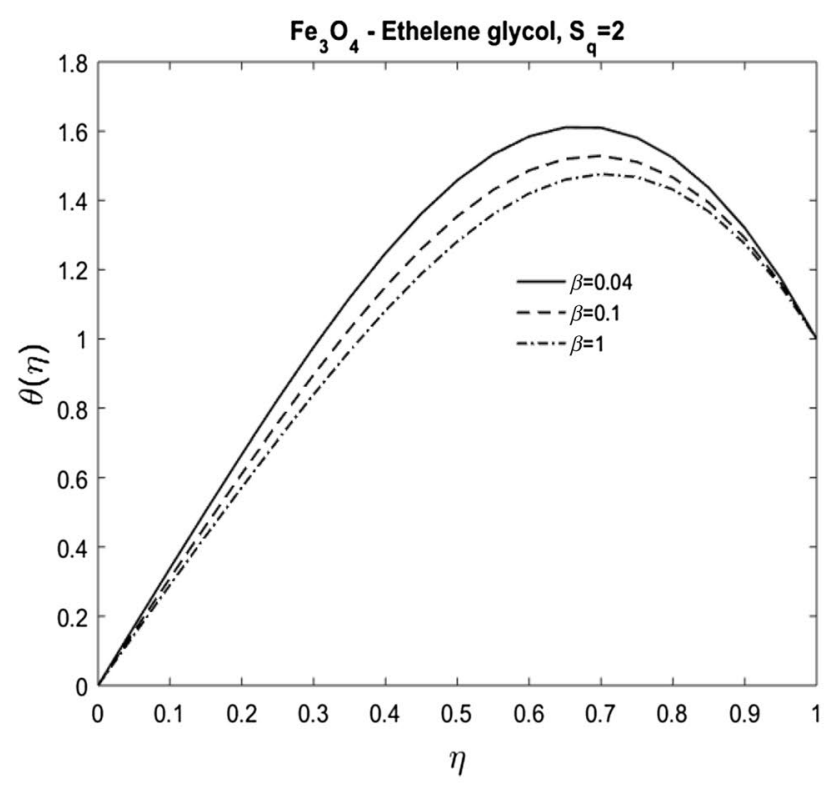

(a)

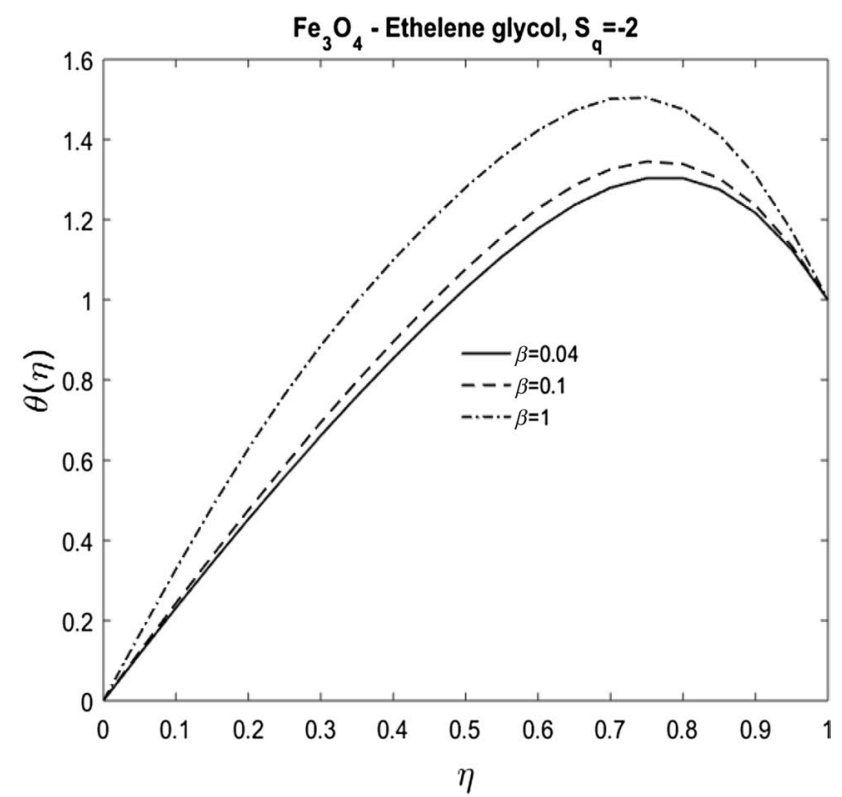

(b)

Fig. 8 Temperature profile for different values of couple stress parameter 


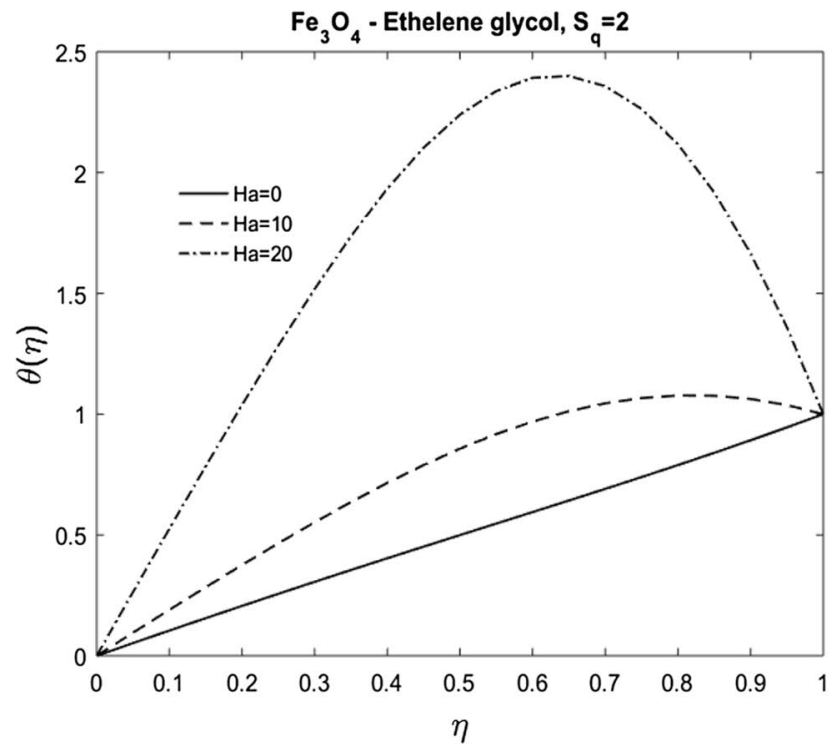

(a)

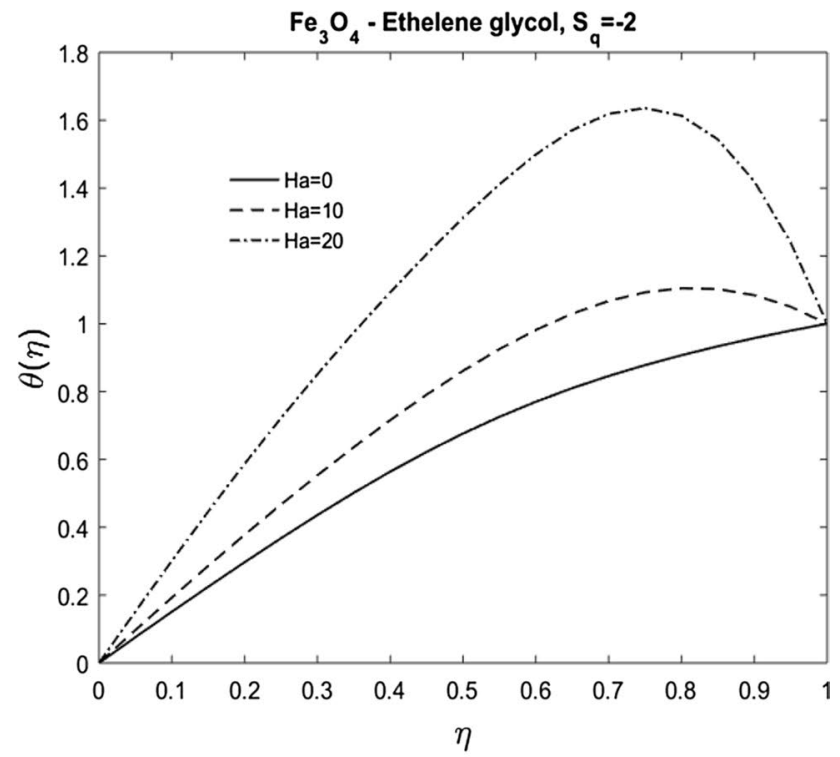

(b)

Fig. 9 Temperature profile for different values of Hartmann number

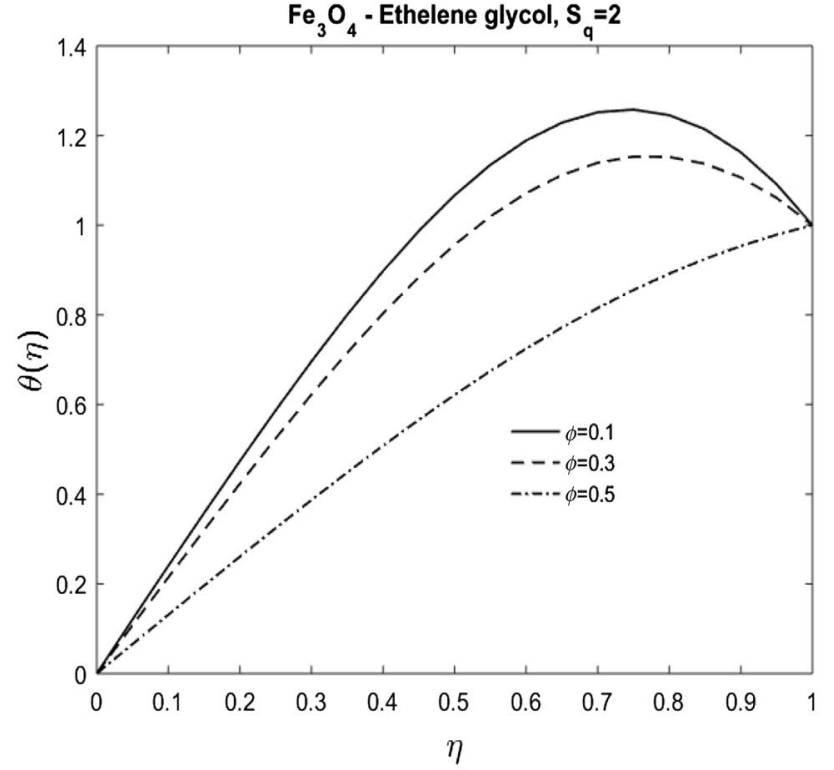

(a)

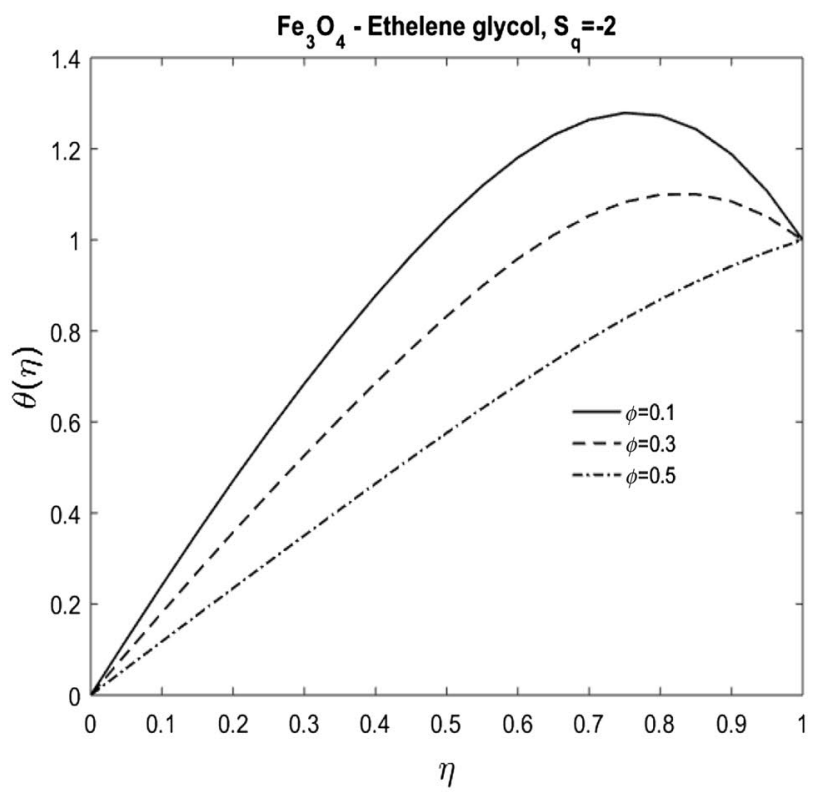

(b)

Fig. 10 Temperature profile for different values of volume fraction 
$\theta(\eta)$ shows a decreasing trend for augmented values of $\varphi$. Also, the thermal boundary layer thickness is more for all increasing values of $\varphi$ when compared to previous temperature profiles. This is due to variation in specific heat and thermal conductivity of $\mathrm{Fe}_{3} \mathrm{O}_{4}$ nanofluid.

\section{Conclusions}

In the present article the analysis of transient hydromagnetic squeezing flow of couple stress $\mathrm{Fe}_{3} \mathrm{O}_{4}$-ethylene glycol nanofluid between two parallel plates has been carried out. The coupled governing non-linear PDE's are transformed to non-linear coupled ODE's by suitable similarity variable and then resolved by using well-known shooting numerical technique. The numerical analysis has been done via graphs and tables to demonstrate the details of the flow features. Based on above discussion few main outcomes of present study are catalogued below.

1. Axial velocity upsurges for augmenting values of couple stress parameter whereas reverse trend is noticed for radial velocity profile.

2. For all augmenting values of $\mathrm{Ha}$ the axial velocity shows the oscillating trend for both case of $\mathrm{S}_{\mathrm{q}}$. However radial velocity increases in both cases of $S_{q}$ for all increasing values of $\mathrm{Ha}$.

3. For all boosting values of volume fraction the axial velocity changes abruptly (greater boundary layer thickness) and radial velocity shows the increasing trend.

4. When $\mathrm{S}_{\mathrm{q}}>0$ the temperature upsurges for augmenting values of $\beta$. However reverse tendency is noticed when $\mathrm{S}_{\mathrm{q}}<0$.

5. For both the cases of $\mathrm{S}_{\mathrm{q}}$ the temperature is increased with Ha whereas scenario is reverse for increasing of volume fraction.

Acknowledgements The authors are thankful to the referees their valuable suggestions which led to improvement of the paper.

\section{Compliance with ethical standards}

Conflict of interest The author(s) declare that they have no competing interests.

\section{References}

1. Choi SUS, Estman JA (1995) Developments and applications of non-Newtonian flows. ASME Publ Fed 231:99
2. Wen D, Ding $Y$ (2004) Experimental investigation into convective heat transfer of nanofluids at the entrance region under laminar flow conditions. Int J Heat Mass Transf 47:5181

3. Eastman JA, Choi SUS, Yu W, Thompson LJ (2001) Anomalously increased effective thermal conductivities of ethylene glycolbased nanofluids containing copper nanoparticles. Appl Phys Lett 78:718

4. Xie H, Lee H, Youn W, Choi M (2004) J Appl Phys 94:4967

5. Minkowycz WJ, Sparrow EM, Abrahim JP (2013) Advances in numerical heat transfer. Nanoparticle Heat Transfer Fluid Flow

6. Wong KV, Leon OD (2010) Applications of nanofluids: current and future. Adv Mech Eng 2010:1

7. Saidur R, Leong KY, Mohammad HA (2011) A review on application and challenges of nanofluids. Renew Sustain Energy Rev 15:1646

8. Parekh K, Lee HS (2010) Magnetic field induced enhancement in thermal conductivity of magnetite nanofluid. J Appl Phys 107(9):09A310

9. Stefan MJ (1874) Sitzungsberichte der Akademie der Wissenschaften in Wien. Mathematik- Naturwissen 69:713

10. Tichy J, Winner WO (1970) Inertial considerations in parallel circular squeeze film bearings. Trans ASME J Lub Technol 92:588

11. Singh P, Radhakrishnan V, Narayan KA (1990) Squeezing flow between parallel plates. Ingenieur-Archiv 60:274

12. Hamdan MH, Baron RM (1992) Analysis of the squeezing flow of dusty fluids. Appl Sci Res 49:345

13. Hamza EA (1999) Suction and injection effects on a similar flow between parallel plates. J Phys D Appl Phys 32:656

14. Duwairi HM, Tashtoush B, Damseh R (2004) Squeezed flow and heat transfer over a porous surface for viscous fluid. Heat Mass Transf 41:112

15. Mahmood M, Asghar S, Hossain MA (2007) Heat Mass Transf 44:165

16. Siddiqui AM, Irum S, Ansari AR (2008) Unsteady squeezing flow of a viscous MHD fluid between parallel plates, a solution using the homotopy perturbation method. Math Model Anal 13(4):565

17. Mustafa M, Hayat T, Obaidat S (2012) On heat and mass transfer in the unsteady squeezing flow between parallel plates. Meccanica 47:1581

18. Petrov AG, Kharlamova IS (2014) The solutions of Navier-Stokes equations in squeezing flow between parallel plates. Eur J Mech B 48:40

19. Qayyum A, Awais M, Alsaedi A, Hayat T (2012) Unsteady squeezing flow of Jeffery fluid between two parallel disks. Chin Phys Lett 29(3):034701

20. Khan U, Khan SK, Ahmed N, Bano S, Mohyud-Din ST (2016) Heat transfer analysis for squeezing flow of a Casson fluid between parallel plates. Ain Shams Eng J 7:497

21. Vijayakumar B, Kesavan S (2017) Non-newtonian effects of incompressible second order fluid on equilateral triangular plates. Int J Pure Appl Math 113(13):301-309

22. Lin Jaw-Ren, Chu Li-Ming, Liang Long-Jin (2013) Effects of viscosity-pressure dependency on the non-Newtonian squeeze film of parallel circular plates. Lubr Sci 25:1

23. Naduvinamani NB, Siddangouda A, Ayyappa GH (2016) Tribology 10(3):126

24. Sherwood JD (2011) Squeeze flow of a power-law fluid between non-parallel plates. J Non-Newtonian Fluid Mech $166: 289$

25. Kashyap KP, Kumar NN (2019) Squeezing flow of a chemically reacting upper convected Maxwell nanofluid with slip effects. J Nanofluids 8:509

26. Stokes VK (1966) Couple stresses in fluids. Phys Fluids 9:1709 
27. Lakshmana Rao SK, lyengar TKV (1985) Analytical and computational studies in couple stress fluid flows. UGC Research Project: C-8-4/82 SR III

28. Naduvinamani NB, Hiremath PS, Gurubasavaraj G (2001) Squeeze film lubrication of a short porous journal bearing with couple stress fluids. Tribol Int 34(11):739

29. Adesanya Samuel O, Makinde Oluwole D (2015) Irreversibility analysis in a couple stress film flow along an inclined heated plate with adiabatic free surface. Phys A 432:222

30. Govindarajan A, Siva EP, Vidhya M (2015) Combined effect of heat and mass transfer on MHD Peristaltic transport of a couple stress fluid in a inclined asymmetric channel through a porous medium. Int J Pure Appl Math 105:685

31. Lin JR, Hung CR (2007) Combined effects of non-Newtonian couple stresses and fluid inertia on the squeeze film characteristics between a long cylinder and an infinite plate. Fluid Dyn Res 39:616

32. Grimm RJ (1976) Squeezing flows of Newtonian liquid films an analysis including fluid inertia. Appl Sci Res 32:149

33. Hamza EA (1988) Unsteady MHD squeezing flow between two parallel rotating discs. J Tribol 110:375

34. Bhattacharyya S, Pal A (1997) Unsteady MHD squeezing flow between two parallel rotating discs. Mech Res Commun 24:615

35. Ran XJ, Zhu QY, Li Y (2007) Approximate analysis of MHD squeeze flow between two parallel disks with suction or injection by homotopy perturbation method. Commun Nonlinear Sci Numer Simul 8:179

36. Domairry G, Aziz A (2009) Approximate analysis of MHD squeeze flow between two parallel disks with suction or injection by homotopy perturbation method. Math Probl Eng 2009:1

37. Hussain A, Mohyud-din ST, Cheema TA (2012) Analytical and numerical approaches to squeezing flow and heat transfer between two parallel disks with velocity slip and temperature jump. Chin Phys Lett 29:114705

38. Khan U, Ahmed N, Zaidi ZA, Asadullah S, Mohyud-Din ST (2014) MHD squeezing flow between two infinite plates. Ain Shams Eng J. 5:187
39. Qayyum M, Khan H, Khan O (2017) Slip analysis at fluid-solid interface in MHD squeezing flow of Casson fluid through porous medium. Result Phys 7:732-750

40. Ghadikolaei SS, Hosseinzadeh K, Ganji DD (2017) Analysis of unsteady MHD Eyring-Powell squeezing flow in stretching channel with considering thermal radiation and Joule heating effect using AGM. Case Stud Therm Eng 10:579-594

41. Muhammad T, Hayat T, Alsaedi A, Qayyum A (2017) Hydromagnetic unsteady squeezing flow of Jeffrey fluid between two parallel plates. Chin J Phys 55:1511

42. Adesanya SO, Ogunseye HA, Jangili S (2018) Unsteady squeezing flow of a radiative Eyring-Powell fluid channel flow with chemical reactions. Int J Therm Sci 125:440

43. Hayat T, Sajjad R, Alsaedi A, Muhammad T, Ellahi R (2017) On squeezed flow of couple stress nanofluid between two parallel plates. Result Phys 7:553

44. Nagler J (2016) The electroosmotic magnetohydrodynamic nano flow. J Nanofluids 5(6):898

45. Ahmad S, Khan MI, Hayat T, Khan MI, Alsaedi A (2018) Entropy generation optimization and unsteady squeezing flow of viscous fluid with five different shapes of nanoparticles. Colloids Surf A 554:197

46. Brinkman HC (1952) The viscosity of concentrated suspensions and solutions. J Chem Phys 20:571

47. Tiwari RK, Das MK (2007) Heat transfer augmentation in a twosided lid-driven differentially heated square cavity utilizing nanofluids. Int J Heat Mass Trans 50:2002

48. Khanafer K, Vafai K, Lightstone M (2003) Buoyancy-driven heat transfer enhancement in a two-dimensional enclosure utilizing nanofluids. Int J Heat Mass Trans 46:3639

49. Maxwell JC (1904) A treatise on electricity and magnetism, 2nd edn. Oxford University Press, Cambridge

Publisher's Note Springer Nature remains neutral with regard to jurisdictional claims in published maps and institutional affiliations. 